\title{
The predictive value of serum concentrations of anti-Müllerian hormone for oocyte quality, fertilization, and implantation
}

\author{
Edson Borges ${ }^{1,2}$, Daniela P. A. F. Braga ${ }^{1,2,3}$, Amanda Setti ${ }^{1,2}$, Rita de Cássia Figueira' ${ }^{1}$, Assumpto Iaconelli Jr ${ }^{1,2}$ \\ ${ }^{1}$ Fertility Medical Group, São Paulo, SP - Brazil \\ ${ }^{2}$ Instituto Sapientiae - Centro de Estudos e Pesquisa em Reprodução Assistida, São Paulo, SP - Brazil \\ 3 Disciplina de Urologia, Área de Reprodução Humana, Departamento de Cirurgia, Universidade Federal de São \\ Paulo, São Paulo, SP - Brazil
}

\begin{abstract}
Objective: This study aimed to identify a possible correlation between serum levels of anti-Müllerian hormone (AMH) and oocyte quality, embryo developmental competence, and implantation potential.

Methods: 4488 oocytes obtained from 408 patients undergoing ICSI cycles were evaluated. Oocyte dimorphisms, embryo quality on days two and three, blastocyst formation competence, fertilization rates, implantation rates, and pregnancy rates were correlated with serum levels of AMH using Pearson's correlation coefficient and regression analysis.

Results: A positive correlation was observed between serum levels of $\mathrm{AMH}$ and number of retrieved oocytes (CC: $0.600, p<0.001)$, fertilization rate (CC:0.595, $p=0.048)$, and number of obtained embryos (CC:0.495, $p<0.001$ ). $\mathrm{AMH}$ did not affect the quality of cleavage stage embryos or the chance of blastocyst formation. However, AMH levels affected oocyte quality (OR:0.75, CI 0.44-0.96, $p<0.001$ ), and implantation (CC:0,116, $p=0.031$ ) and pregnancy (OR:1.22, CI:1.03-1.53, $p<0.001$ ) rates.

Conclusion: Serum levels of $\mathrm{AMH}$ are a useful predictor of ovarian response to COS, oocyte quality, and fertilization. However, AMH levels may also compromise clinical outcomes; lower AMH levels did not impair embryo development.
\end{abstract}

Keywords: Oocyte, blastocyst, embryo implantation, pregnancy, COS cells, anti-Müllerian hormone

\section{INTRODUCTION}

Anti-Müllerian hormone (AMH), also known as Müllerian inhibiting substance, is a peptide growth factor and a member of the tissue growth factor beta superfamily. In male fetuses, the development of the internal reproductive tract requires the presence of $\mathrm{AMH}$ to cause the involution of the Müllerian ducts, the anlagen of the female reproductive tract (Nussey \& Whitehead, 2001). In females, fetal AMH is produced after 36 weeks of gestation by the granulosa cells of primary, secondary, pre-antral, and early antral follicles (Durlinger et al., 1999; Rajpert-De Meyts et al., 1999; Weenen et al. 2004), and it continues to be expressed until the follicles reach the antral phase and are more than $2 \mathrm{~mm}$ in diameter (Messinis, 2006).

It has been suggested that $\mathrm{AMH}$ inhibits the initiation of primordial follicle growth from the ovarian reserve (Weenen et al., 2004; Nilsson et al., 2007). In mice, it decreases the sensitivity of follicles to $\mathrm{FSH}$, thereby inhibiting $\mathrm{FSH}$-induced antral follicle growth (Durlinger et al., 2002). Although not fully understood, AMH may play a role in regulating the recruitment of cohorts of antral follicles (reviewed in (Baerwald et al., 2012).

The anti-Müllerian hormone, barely detectable at birth in females, peaks after puberty (Rajpert-De Meyts et al., 1999). In line with this, AMH levels have shown greater sensitivity to ovarian aging (de Vet et al., 2002) and a stronger relationship with the number of early antral follicles (Fanchin et al., 2003). In fact, the serum level of AMH has been established as the most valuable marker of ovarian reserve, as it correlates highly with the baseline antral follicle count. In addition, AMH levels are a useful predictor of ovarian response to controlled ovarian stimulation (COS) (van Rooij et al., 2002; Eldar-Geva et al., 2005; Tremellen et al., 2005; Nakhuda et al., 2006; Nakhuda et al., 2007; Nardo et al., 2009).

Previous studies found that, besides ovarian response to COS, the serum level of $\mathrm{AMH}$ can be used as a predictor of severity of endometriosis (Shebl et al., 2009). Anti-Müllerian hormone measurements also offer relatively high specificity and sensitivity as a diagnostic marker for polycystic ovary syndrome (Pigny et al., 2006). Moreover, as AMH declines with age and continues to decline until it reaches a stage in which it becomes undetectable in serum after menopause, it has also been suggested that a single $\mathrm{AMH}$ measurement may be a good predictor of the onset of menopause in aging women.

Despite being a good marker of ovarian response, $\mathrm{AMH}$ fails to predict the outcome of pregnancy (Lie Fong et al., 2008; Talebian et al., 2008; Riggs et al., 2011), since, as suggested by Loh \& Maheshwari (2011), it cannot predict the quality of the oocyte. However, previously published reports have focused on the assessment of embryo quality (Hazout et al., 2005; Silberstein et al., 2006; Smeenk et al., 2007; Lie Fong et al., 2008; Talebian et al., 2008) rather than oocyte quality, considering specifically intracytoplasmic and extracytoplasmic oocyte defects.

In fact, the number of retrieved oocytes and the quality of oocytes and embryos significantly impact the outcomes of assisted reproductive technology (ART). It has been reported that, although the percentage of embryo transfers that leads to deliveries has steadily increased, in the last years, the vast majority of embryos that are produced in vitro fail to develop into an infant (Kovalevsky \& Patrizio, 2005). Therefore, the goal of the present study was to identify a possible correlation between serum levels of $\mathrm{AMH}$ and the following: (i) oocyte quality, (ii) embryo developmental competence, and (iii) implantation potential.

\section{MATERIALS AND METHOD \\ Study Design}

This study analyzed the correlation between serum levels of AMH and (i) maternal age, (ii) maternal BMI, (iii) FSH dose for ovarian stimulation, (iv) number of aspirated follicles, (v) number of retrieved oocytes, (vi) number of retrieved MII mature oocytes, (vii) oocyte quality, (viii) fertilization rate, (ix) number of obtained embryos, (x) 
number of obtained high-quality embryos, (xi) embryo quality on day two, (xii) embryo quality on day three, (xiii) chance of blastocyst formation, (xiv) number of transferred embryos, (xv) implantation rate, and (xvi) pregnancy rate.

Intra and extracytoplasmic defects were recorded before sperm injection for purposes of oocyte quality assessment. In embryo quality evaluation, the embryos were categorized as high or low quality on days two, three, and five. Fertilization rate was defined based on the number of oocytes presenting two clearly distinct pronuclei 18 hours after ICSI divided by the number of injected oocytes. Implantation rate was calculated as the number of gestational sacs divided by the number of embryos transferred per patient. Clinical pregnancy was defined by the presence of a gestational sac with heartbeat viewed on ultrasound examination 4-6 weeks after embryo transfer.

The assessment included 4488 oocytes obtained from 408 patients undergoing ICSI cycles between January 2011 and August 2013. Serum AMH tests were included as a standard measure in the IVF program. Tests were run prior to the start of each cycle and $\mathrm{AMH}$ levels were recorded. The oocytes were evaluated immediately before sperm injection, and the embryos were evaluated $16-18 \mathrm{~h}$ post-ICSI and on days two, three and five of development.

Inclusion criteria were as follows: women of good physical and mental health, with regular menstrual cycles of 25-35 days, normal basal FSH and LH levels and BMI lower than $30 \mathrm{~kg} / \mathrm{m}^{2}$, presence of both ovaries and an intact uterus, undergoing the first or second ICSI cycle. Patients with endometriosis or gynecological/medical disorders and a negative result in a screening for sexually transmitted diseases were excluded. All cases of severe alteration in spermatogenesis, including frozen and surgically retrieved sperm, were also excluded from the study.

The implantation rate was defined as the total number of gestational sacs divided by the total number of transferred embryos. Clinical pregnancy was defined as the presence of a gestational sac viewed on ultrasound examination 4-6 weeks after embryo transfer.

The patients gave written consent before joining the study and agreed to share the outcomes of their cycles for research purposes. The local review board approved the study.

\section{Controlled ovarian stimulation}

Controlled ovarian stimulation was achieved using a daily dose of recombinant FSH (Gonal-F; Serono, Geneva, Switzerland) starting on day three of the cycle. Pituitary down-regulation was performed using a GnRH antagonist (Cetrotide, Serono, Geneva, Switzerland), started when at least one follicle $\geq 14 \mathrm{~mm}$ was viewed.

Follicular growth was monitored using transvaginal ultrasound examination starting on day four of gonadotropin administration. When adequate follicular growth and serum E2 levels were observed, recombinant hCG (Ovidrel; Serono, Geneva, Switzerland) was administered to trigger final follicular maturation. The oocytes were collected 35 hours after hCG administration through transvaginal ultrasound-guided ovum pick-up.

\section{Preparation of oocytes}

The retrieved oocytes were maintained in culture medium (Global ${ }^{\circledR}$ for fertilisation, LifeGlobal, Connecticut, USA) supplemented with $10 \%$ protein supplement (LGPS, LifeGlobal, Connecticut, USA) and covered with paraffin oil (Paraffin oil P.G., LifeGlobal, Connecticut, USA) for two to three hours before the removal of cumulus cells. The surrounding cumulus cells were removed after exposure to a HEPES-buffered medium containing hyaluronidase (80IU/ $\mathrm{mL}$, LifeGlobal, Connecticut, USA). The remaining cumulus cells were gently removed with a hand-drawn Pasteur pipette (Humagen Fertility Diagnostics, Charlottesville, USA).

Oocyte morphology was assessed immediately before sperm injection (4 hours after retrieval) using an inverted Nikon Diaphot microscope (Eclipse TE 300; Nikon ${ }^{\circledR}$, Tokyo, Japan) with a Hoffmann modulation contrast system under 400X magnification. The following oocyte dysmorphisms were recorded: intra-cytoplasmic defects such as (i) cytoplasm color, (ii) vacuoles in the ooplasm, (iii) aggregates of smooth endoplasmic reticulum clusters (ERC) in the ooplasm and (iv) retractile bodies; and extracytoplasmic defects such as (i) large perivitelline space (PVS), (ii) PVS granularity, (iii) zona pellucida (ZP) abnormalities, (iv) shape abnormalities, and ( $v$ ) fragmented polar body (PB).

Oocytes releasing the first polar body were considered mature and were used for ICSI.

\section{Intracytoplasmic sperm injection}

Intracytoplasmic sperm injection was performed in a microinjection dish prepared with $4-\mu L$ droplets of buffered medium (Global ${ }^{\circledR}$ W/HEPES, LifeGlobal, Connecticut, USA) and covered with paraffin oil on the heated stage of an inverted microscope $\left(37.0 \pm 0.5^{\circ} \mathrm{C}\right)$. Approximately 16 hours after ICSI, fertilization was confirmed by the presence of two pronuclei and the extrusion of the second polar body. Embryos were maintained in a 50- $\mu \mathrm{L}$ drop of culture medium (Global ${ }^{\circledR}$, LifeGlobal, Connecticut, USA) supplemented with $10 \%$ protein supplement and covered with paraffin oil in a humidified atmosphere under $6 \% \mathrm{CO}_{2}$ at $37^{\circ} \mathrm{C}$ for three days.

\section{Embryo morphology evaluation}

Embryo morphology was assessed $16-18 \mathrm{~h}$ post-ICSI and on the mornings of days two, three and five of embryo development using an inverted Nikon Diaphot microscope (Eclipse TE 300; Nikon, Tokyo, Japan) with a Hoffmann modulation contrast system under 400X magnification.

Cleavage stage morphology was assessed based on the following parameters: number of blastomeres, percent fragmentation, variation in blastomere symmetry, presence of multinucleation, and defects in the zona pellucida and cytoplasm. High-quality cleavage stage embryos had to show the following characteristics: 4 cells on day two or $8-10$ cells on day three, $<15 \%$ fragmentation, symmetric blastomeres, absence of multinucleation, colorless cytoplasm with moderate granulation and no inclusions, absence of perivitelline space granularity and absence of zona pellucida dysmorphism. Embryos lacking any of these characteristics were considered to be of low quality.

In blastocyst formation assessment the embryos were given a numerical score from 1 to 6 based on their degree of expansion and hatching statuses as follows: 1 , an early blastocyst with a blastocoel that was less than half the volume of the embryo; 2, a blastocyst with a blastocoel that was greater than half the volume of the embryo; 3 , a full blastocyst with a blastocoel that completely filled the embryo; 4, an expanded blastocyst; 5, a hatching blastocyst; and 6, a hatched blastocyst. Embryos receiving scores of 3 and greater were considered a full blastocyst.

\section{Blood collection and Anti-Müllerian hormone analysis \\ When the patients visited the outpatient department,} serum AMH levels were obtained, regardless of the menstrual phase, using a human AMH ELISA Kit (CUSABIO, Wuhan, China).

Blood samples were collected by peripheral venipuncture and centrifuged at $1000 \times \mathrm{g}, 4^{\circ} \mathrm{C}$, for 15 minutes, within 30 
minutes of collection; the plasma portion was removed and assayed.

The human AMH ELISA Kits were used according to supplier instructions. The intra- and inter-assay coefficients of variation for all assays were $<10$ and $15 \%$, respectively. Limits of detection were: $1 \mathrm{ng} / \mathrm{mL}-75 \mathrm{ng} / \mathrm{mL}$.

\section{Statistical analysis}

Cycle and patient characteristics were expressed as the mean \pm standard deviation for continuous variables; percentages were used for categorical variables.

Pearson's correlation coefficient was used to evaluate the relationship between serum AMH levels and continuous variables. The results were expressed in the form of correlation coefficients (CC) and $p$-values.

Binary regression analysis was performed to evaluate the influence of AMH levels on pregnancy rates, embryo quality, blastocyst formation, and oocyte defects. These regressions were adjusted for female age, total level of FSH used in controlled ovarian stimulation, and the initial dose of $\mathrm{FSH}$, as these are potentially confounding factors in the association between AMH level and the evaluated variables. The results were expressed as odds ratios (OR), $95 \%$ confidence intervals (CI) and $p$-values.

Significance was assigned to events with a $p$-value $<0.05$. Data analysis was carried out on Minitab Statistical Software (version 14).

\section{RESULTS}

Patient and cycle characteristics were as follows: maternal age: $37.7 \pm 5.4(20-43)$ years, paternal age: $39.0 \pm 6.7$ (24-65) years; body mass index (BMI): $24.4 \pm 3.9$ (14.2-37.2); total dose of FSH administered in ovarian stimulation: $2370.9 \pm 855.3$ (1900-3450)IU; number of aspirated follicles: $8.0 \pm 10.5$ (1-20); number of retrieved oocytes: $6.7 \pm 7.4(1-25)$; number of retrieved mature oocytes: $5.9 \pm 6.2$ (1-18); fertilization rate: $82.7 \pm 20.8 \%$ (10-100); embryos obtained: $4.5 \pm 5.5$ (1-16); high-quality embryos obtained: $1.5 \pm 1.1(1-5)$; embryos transferred: $1.0 \pm 1.8(1-3)$; implantation rate (\%): $22.0 \pm 40.2(0-100)$; and pregnancy rate: $28.9 \%$.

The causes of infertility were as follows: male factor (146/408:35.8\%), tubal factor (26/408:6.4\%), unexplained infertility (29/408:7.1\%), polycystic ovary syndrome (PCOS, 25/408:6.1\%), female factor (127/408: $31.1 \%)$, and others (55/408:13.5\%).

As expected, an inverse correlation was found between serum AMH levels and maternal age (CC:-0.279, $p<0.001)$. Significant positive correlations were identified between serum $\mathrm{AMH}$ levels and number of aspirated follicles (CC: $0.626, p<0.001$ ), number of retrieved oocytes (CC:0.600, $p<0.001)$, number of mature oocytes (CC:0.588, $p<0.001$ ), fertilization rate (CC:0.595, $p=0.048)$, number of obtained embryos (CC:0.495, $p<0.001$ ), number of high-quality embryos (CC:0.504, $p<0.001$ ), number of transferred embryos (CC:0.221, $p<0.001$ ), and implantation rate (CC:0,116, $p=0.031$ ) (Table 1).

Serum levels of $\mathrm{AMH}$ also impacted pregnancy rates (OR:1.22, CI:1.03-1.53, $p<0.001$, Table 2).

The FSH dose that was used for ovarian stimulation (CC:0.089, $p=0.075$ ) and female BMI (CC:-0.203, $p=0.065$ ) did not correlate with serum AMH levels.

The serum level of AMH did not impact embryo quality on days two or three or the chance of blastocyst formation. However, AMH levels affected oocyte quality (OR:0.75, CI:0.44-0.96, $p<0.001$, Table 3).

The presence of aggregates of smooth ERC (OR:0.82, CI:0.63-0.99, $p=0.025$ ), large PVS (OR:0.84, CI:0.76$0.93, p<0.001$ ), PVS granularity (OR:0.91, CI:0.79-0.96, $p<0.001)$ ) ZP abnormalities (OR:0.85, CI:0.73-0.94, $p<0.001)$, and shape abnormalities (OR:0.83, CI:0.51$0.93, p=0.010$ ) were affected by the serum level of $\mathrm{AMH}$. The presence of other dysmorphisms, such as cytoplasm color, vacuoles in the ooplasm, retractile bodies, and fragmented PB was not affected by the level of AMH (Table $3)$.

\section{DISCUSSION}

Oocyte quality has been regarded as a variable that influences the implantation of derived embryos. It has been previously described that when cumulus cells are denuded for ICSI, more than $60 \%$ of all oocytes show at least one abnormal morphological characteristic (Braga et al., 2013). Considering that both extreme forms of ovarian response (low and hyper-response) may be associated with diminished oocyte quality (van Rooij et al., 2003; Figueira et al., 2011; Braga et al., 2012), we have hypothesized that lower levels of AMH might have a negative effect on oocyte and embryo development. Our results demonstrated that neither embryo quality on the cleavage stage nor the chance of blastocyst formation was correlated with AMH levels. However, the serum level of AMH was positively correlated with oocyte quality.

It remains unclear whether the correlation between $\mathrm{AMH}$ and the presence of oocyte defects is due to decreased secretion by granulosa cells in poor quality oocytes or due to a possible detrimental effect of low levels of AMH on oocyte quality. Indeed, AMH might play an important role in primordial follicle selection and cyclic growing follicle recruitment (Carlsson et al., 2006). Moreover, AMH might regulate the selection of the dominant follicle through the inhibitory effects of $\mathrm{AMH}$ on the initial recruitment of primary follicles from the resting primordial follicle pool (Durlinger et al., 2001) and through the regulation of FSH sensitivity in the human ovary (Kevenaar et al., 2007).

Our data also support previous reports on the prognostic value of AMH levels on female age (Broer et al., 2009; Nelson et al., 2011) and ovarian response (Broer et al., 2009). However, the inability to estimate embryo quality and blastocyst formation competence by AMH levels disagreed with the work of Silberstein et al. (2006), in which a relationship was reported between AMH levels and embryo morphology. In the mentioned study, the morphological assessment of cleavage stage embryos consisted of the evaluation of cell number, percent fragmentation, and blastomere symmetry. The presence of multinucleation and defects in the zona pellucida and cytoplasm was not evaluated. Moreover, it failed to define high/low-quality embryos or to adopt any form of embryo scoring criteria.

In our study, the serum level of $\mathrm{AMH}$ affected the presence of intra- and extracytoplasmic defects. This finding agreed with an elegant trial from Fanchin et al. (2007), in which a clinical model of monodominant follicle in vitro fertilization to determine whether $\mathrm{AMH}$ production by a single, preovulatory follicle, as assessed by follicular fluid AMH measurements, was positively related to oocyte and embryo development. A direct link was suggested between the aptitude of granulosa cells to produce AMH and the functional quality of the oocyte, as reflected by its competence to become an embryo with implantation potential.

Ebner et al. (2006) also demonstrated that serum levels of $\mathrm{AMH}$ were associated with oocyte quality in stimulated cycles. In their study, embryo quality was not estimated using baseline AMH levels, which was also in accordance with our evidence. However, the fertilization rate was not correlated with the level of $\mathrm{AMH}$, which disagreed with the results of the present study. 


\begin{tabular}{|c|c|c|c|}
\hline \multicolumn{4}{|l|}{$\begin{array}{l}\text { Table 1. Pears } \\
\text { characteristics. }\end{array}$} \\
\hline \multirow{12}{*}{$\begin{array}{l}\text { Serum level of } \\
\text { AMH }\end{array}$} & Variable & Correlation coefficient & $\boldsymbol{p}$ \\
\hline & Maternal age & -0.279 & $<0.001$ \\
\hline & Maternal BMI & -0.203 & 0.065 \\
\hline & FSH dose & 0.089 & 0.075 \\
\hline & Number of aspirated follicles & 0.626 & $<0.001$ \\
\hline & Number of retrieved oocytes & 0.600 & $<0.001$ \\
\hline & Number of retrieved MII mature oocytes & 0.588 & $<0.001$ \\
\hline & Fertilization Rate & 0.595 & 0.048 \\
\hline & Number of obtained embryos & 0.495 & $<0.001$ \\
\hline & Number of obtained high-quality embryos & 0.504 & $<0.001$ \\
\hline & Number of transferred Embryos & 0.221 & $<0.001$ \\
\hline & Implantation Rate & 0.116 & 0.031 \\
\hline
\end{tabular}

AMH: Anti-Müllerian hormone.

Table 2. Binary regression analysis of the effect of the serum level of Anti-Müllerian hormone on embryo quality on days
two or three, chance of blastocyst formation, and pregnancy odds
\begin{tabular}{|l|l|c|c|c|c|}
\hline \multirow{2}{*}{ Predictor variable } & Response variable & $\boldsymbol{P}$ & OR & CI: Lower & CI: Upper \\
\hline & Embryo quality on day two & 0.846 & 1.01 & 0.97 & 1.03 \\
\cline { 2 - 6 } Serum level of AMH & Embryo quality on day three & 0.724 & 0.99 & 0.73 & 2.19 \\
\cline { 2 - 7 } & Blastocyst formation & 0.574 & 0.98 & 0.54 & 1.96 \\
\cline { 2 - 7 } & Pregnancy & $\mathbf{< 0 . 0 0 1}$ & 1.22 & $1.03-$ & 1.53 \\
\hline
\end{tabular}

AMH: Anti-Müllerian hormone.

Table 3. Binary regression analysis of oocyte dysmorphisms affected by the serum level of Anti-Müllerian hormone

\begin{tabular}{|c|c|c|c|c|c|}
\hline Predictor variable & Response variable & $\boldsymbol{p}$ & OR & CI: Lower & CI: Upper \\
\hline \multirow{10}{*}{ Serum level of AMH } & At least one oocyte defect & $<0.001$ & 0.75 & 0.44 & 0.96 \\
\hline & Cytoplasm color & 0.056 & 0.79 & 0.65 & 1.03 \\
\hline & Vacuoles in the ooplasm & 0.465 & 0.97 & 0.91 & 1.09 \\
\hline & Aggregates of smooth ERC & 0.025 & 0.82 & 0.63 & 0.99 \\
\hline & Retractile Bodies & 0.256 & 0.83 & 0.53 & 1.53 \\
\hline & Large PVS & $<0.001$ & 0.84 & 0.76 & 0.93 \\
\hline & PVS granularity & $<0.001$ & 0.91 & 0.79 & 0.96 \\
\hline & ZP abnormalities & $<0.001$ & 0.85 & 0.73 & 0.94 \\
\hline & Shape abnormalities. & 0.010 & 0.83 & 0.51 & 0.93 \\
\hline & Fragmented PB & 0.456 & 0.99 & 0.86 & 1.09 \\
\hline
\end{tabular}

AMH: Anti-Müllerian hormone, ERC: Endoplasmic reticulum cluster, PVS: perivitelline space, ZP: Zona pellucida, PB: Polar Body.

The process of fertilization is initiated by gamete fusion and can be considered to include all the events associated with egg activation until the time the egg is committed to embryonic development (Nixon et al., 2000). The observation that the level of AMH negatively affected fertilization and oocyte quality suggests a possible relationship between granulosa cell metabolism and oocyte developmental competence. Previous studies have shown that the degree of apoptosis of mural and cumulus granulosa cells negatively affects the developmental competence of the oocyte (Nakahara et al., 1997; Zeuner et al., 2003). Accordingly, human (Weenen et al., 2004) and animal (Bézard et al., 1987; Baarends et al., 1995) follicular atresia prevents the expression of $\mathrm{AMH}$.

In this study, it is unclear why $A M H$ serum levels affected fertilization rates and oocyte quality, but not embryo development. It may be suggested that, although the level of AMH has a significant negative effect on oocyte quality and fertilization capacity, once fertilized, oocytes do not show compromised developmental competence in vitro. Embryo genome is known to begin express between the four- and eight-cell stages of human embryo development. At this stage, the sperm-derived genes linked to embryo viability have also been disrupted (Tesarík et al., 1988). 
In addition to fertilization, our results showed the existence of a relationship between level of $\mathrm{AMH}$ and chance of pregnancy. Loh \& Maheshwari (2011) previously described that $\mathrm{AMH}$ fails to predict the odds of pregnancy. Nonetheless, some reported that extremely low levels of AMH are associated with non-pregnancy (La Marca et al., 2011) while others described moderate to reasonable pregnancy rates following extremely low levels of serum AMH (Lamazou et al., 2011; Weghofer et al., 2011). In our study, the chance of pregnancy and the number of obtained embryos, high-quality embryos, and transferred embryos were positively correlated with the level of AMH. Therefore, it might be argued that the correlation between $\mathrm{AMH}$ and pregnancy depends on the number of obtained oocytes and embryos available for transfer, rather than embryo quality. On the other hand, AMH levels also negatively impacted the implantation rate, which measures the number of transferred embryos.

These findings raised the question as to whether the worse outcomes observed for patients with lower AMH levels correlated with lower response to COS, decreased oocyte quality or both.

In conclusion, our findings indicated that the serum level of $\mathrm{AMH}$ is a useful predictor of ovarian response to COS, oocyte quality, fertilization, and implantation. However, although AMH levels might compromise pregnancy outcomes, lower levels of AMH do not impair the embryo developmental competence.

\section{CONFLICT OF INTERESTS}

The authors have no conflicts of interest to report.

\section{Corresponding author:}

Daniela Braga

Fertility Medical Group São Paulo/SP - Brazil

São Paulo, SP, Brazil.

E-mail: dbraga@fertility.com.br

\section{REFERENCES}

Baarends WM, Uilenbroek JT, Kramer P, Hoogerbrugge JW, van Leeuwen EC, Themmen AP, Grootegoed JA. Anti-müllerian hormone and anti-müllerian hormone type II receptor messenger ribonucleic acid expression in rat ovaries during postnatal development, the estrous cycle, and gonadotropin-induced follicle growth. Endocrinology. 1995;136:4951-62. PMID: 7588229 DOI: $10.1210 /$ endo.136.11.7588229

Baerwald AR, Adams GP, Pierson RA. Ovarian antral folliculogenesis during the human menstrual cycle: a review. Hum Reprod Update. 2012;18:73-91. PMID: 22068695 DOI: 10.1093/humupd/dmr039

Bézard J, Vigier $B$, Tran $D$, Mauléon $P$, Josso $N$. Immunocytochemical study of anti-Müllerian hormone in sheep ovarian follicles during fetal and post-natal development. J Reprod Fertil. 1987;80:509-16. PMID: 3309279

Braga DP, Setti AS, Figueira Rde C, Iaconelli A Jr, Borges E Jr. Contributing factors for the incidence of aneuploidy in older patients undergoing intracytoplasmic sperm injection cycles. J Assist Reprod Genet. 2012;29:911-6. PMID: 22644632 DOI: $10.1007 / s 10815-012-9795-x$
Braga DP, Setti AS, Figueira Rde C, Machado RB, Iaconelli A Jr, Borges $\mathrm{E}$ Jr. Influence of oocyte dysmorphisms on blastocyst formation and quality. Fertil Steril. 2013;100:748-54. PMID: 23768986 DOI: 10.1016/j.fertnstert.2013.05.021

Broer SL, Mol BW, Hendriks D, Broekmans FJ. The role of antimullerian hormone in prediction of outcome after IVF: comparison with the antral follicle count. Fertil Steril. 2009;91:705-14. PMID: 18321493 DOI: $10.1016 /$ j.fertnstert.2007.12.013

Carlsson IB, Scott JE, Visser JA, Ritvos O, Themmen AP, Hovatta O. Anti-Müllerian hormone inhibits initiation of growth of human primordial ovarian follicles in vitro. Hum Reprod. 2006;21:2223-7. PMID: 16720622 DOI: $10.1093 /$ humrep/del165

de Vet A, Laven JS, de Jong FH, Themmen AP, Fauser BC. Antimüllerian hormone serum levels: a putative marker for ovarian aging. Fertil Steril. 2002;77:357-62. PMID: 11821097 DOI: 10.1016/S0015-0282(01)02993-4

Durlinger AL, Gruijters MJ, Kramer P, Karels B, Kumar TR, Matzuk MM, Rose UM, de Jong FH, Uilenbroek JT, Grootegoed JA, Themmen AP. Anti-Müllerian hormone attenuates the effects of FSH on follicle development in the mouse ovary. Endocrinology. 2001;142:4891-9. PMID: 11606457 DOI: $10.1210 /$ endo.142.11.8486

Durlinger AL, Kramer P, Karels B, de Jong FH, Uilenbroek JT, Grootegoed JA, Themmen AP. Control of primordial follicle recruitment by anti-Müllerian hormone in the mouse ovary. Endocrinology. 1999;140:5789-96. PMID: 10579345 DOI: 10.1210/endo.140.12.7204

Durlinger AL, Visser JA, Themmen AP. Regulation of ovarian function: the role of anti-Müllerian hormone. Reproduction. 2002;124:601-9. PMID: 12416998 DOI: $10.1530 /$ rep.0.1240601

Ebner T, Sommergruber M, Moser M, Shebl O, SchreierLechner E, Tews G. Basal level of anti-Müllerian hormone is associated with oocyte quality in stimulated cycles. Hum Reprod. 2006;21:2022-6. PMID: 16679324 DOI: $10.1093 /$ humrep/del127

Eldar-Geva T, Ben-Chetrit A, Spitz IM, Rabinowitz R, Markowitz E, Mimoni T, Gal M, Zylber-Haran E, Margalioth EJ. Dynamic assays of inhibin B, anti-Mullerian hormone and estradiol following FSH stimulation and ovarian ultrasonography as predictors of IVF outcome. Hum Reprod. 2005;20:3178-83. PMID: 16113044 DOI: $10.1093 /$ humrep/dei203

Fanchin R, Mendez Lozano DH, Frydman N, Gougeon A, di Clemente N, Frydman R, Taieb J. Anti-Müllerian hormone concentrations in the follicular fluid of the preovulatory follicle are predictive of the implantation potential of the ensuing embryo obtained by in vitro fertilization. J Clin Endocrinol Metab. 2007;92:1796-802. PMID: 17327387 DOI: $10.1210 /$ jc. 2006-1053

Fanchin R, Schonäuer LM, Righini C, Guibourdenche J, Frydman R, Taieb J. Serum anti-Müllerian hormone is more strongly related to ovarian follicular status than serum inhibin B, estradiol, FSH and LH on day 3. Hum Reprod. 2003;18:323-7. PMID: 12571168 DOI: $10.1093 /$ humrep/deg042 
Figueira Rde C, Braga DP, Semião-Francisco L, Iaconelli A Jr, Borges E Jr. Oocyte yield and dysmorphisms as indicators of biological efficiency in intracytoplasmic sperm injection cycles. Hum Fertil (Camb). 2011;14:41-7. PMID: 21158694 DOI: $10.3109 / 14647273.2010 .523508$

Hazout A, Cohen Bacrie P, Mendoza R, Mendoza C, Tesarik J. Antimullerian Hormone, Ovarian Reserve and Oocyte Quality in Young Oocyte Donors. Fertil Steril. 2005;84:S251. DOI: 10.1016/j.fertnstert.2005.07.650

Kevenaar ME, Themmen AP, Laven JS, Sonntag B, Fong $S L$, Uitterlinden $A G$, de Jong $F H$, Pols $H A$, Simoni $M$, Visser JA. Anti-Müllerian hormone and anti-Müllerian hormone type II receptor polymorphisms are associated with follicular phase estradiol levels in normo-ovulatory women. Hum Reprod. 2007;22:1547-54. PMID: 17337470 DOI: $10.1093 /$ humrep/dem036

Kovalevsky G, Patrizio P. High rates of embryo wastage with use of assisted reproductive technology: a look at the trends between 1995 and 2001 in the United States. Fertil Steril. 2005;84:325-30. PMID: 16084872 DOI: $10.1016 /$ j.fertnstert.2005.04.020

La Marca A, Nelson SM, Sighinolfi G, Manno M, Baraldi E, Roli L, Xella S, Marsella T, Tagliasacchi D, D'Amico $\mathrm{R}$, Volpe A. Anti-Müllerian hormone-based prediction model for a live birth in assisted reproduction. Reprod Biomed Online. 2011;22:341-9. PMID: 21317041 DOI: $10.1016 /$ j.rbmo.2010.11.005

Lamazou F, Genro V, Fuchs F, Grynberg M, Gallot V, Achour-Frydman N, Fanchin R, Frydman R. Serum AMH level is not a predictive value for IVF in modified natural cycle: analysis of 342 cycles. J Gynecol Obstet Biol Reprod (Paris). 2011:40:205-10. PMID: 21398054 DOI: 10.1016/j.jgyn.2011.02.002

Lie Fong S, Baart EB, Martini E, Schipper I, Visser JA, Themmen AP, de Jong FH, Fauser BJ, Laven JS. Anti-Müllerian hormone: a marker for oocyte quantity, oocyte quality and embryo quality? Reprod Biomed Online. 2008;16:664-70. PMID: 18492370 DOI: 10.1016/S1472-6483(10)60480-4

Loh JS, Maheshwari A. Anti-Mullerian hormoneis it a crystal ball for predicting ovarian ageing? Hum Reprod. 2011;26:2925-32. PMID: 21849297 DOI: $10.1093 /$ humrep/der271

Messinis IE. From menarche to regular menstruation: endocrinological background. Ann N Y Acad Sci. 2006;1092:49-56. PMID: 17308132 DOI: $10.1196 /$ annals.1365.004

Nakahara K, Saito H, Saito T, Ito M, Ohta N, Takahashi T, Hiroi $M$. The incidence of apoptotic bodies in membrana granulosa can predict prognosis of ova from patients participating in in vitro fertilization programs. Fertil Steril. 1997;68:312-7. PMID: 9240262 DOI: 10.1016/S0015-0282(97)81521-X

Nakhuda GS, Chu MC, Wang JG, Sauer MV, Lobo RA. Elevated serum müllerian-inhibiting substance may be a marker for ovarian hyperstimulation syndrome in normal women undergoing in vitro fertilization. Fertil Steril. 2006;85:15413. PMID: 16566934 DOI: 10.1016/j.fertnstert.2005.10.052
Nakhuda GS, Sauer MV, Wang JG, Ferin M, Lobo RA. Müllerian inhibiting substance is an accurate marker of ovarian response in women of advanced reproductive age undergoing IVF. Reprod Biomed Online. 2007;14:450-4. PMID: 17425826 DOI: 10.1016/S1472-6483(10)60892-9

Nardo LG, Gelbaya TA, Wilkinson $H$, Roberts SA, Yates A, Pemberton P, Laing I. Circulating basal anti-Müllerian hormone levels as predictor of ovarian response in women undergoing ovarian stimulation for in vitro fertilization. Fertil Steril. 2009;92:1586-93. PMID: 18930213 DOI: $10.1016 /$ j.fertnstert.2008.08.127

Nelson SM, Messow MC, McConnachie A, Wallace H, Kelsey T, Fleming R, Anderson RA, Leader B. External validation of nomogram for the decline in serum anti-Müllerian hormone in women: a population study of 15,834 infertility patients. Reprod Biomed Online. 2011;23:204-6. PMID: 21683652 DOI: $10.1016 /$ j.rbmo.2011.05.006

Nilsson E, Rogers N, Skinner MK. Actions of anti-Mullerian hormone on the ovarian transcriptome to inhibit primordial to primary follicle transition. Reproduction. 2007;134:20921. PMID: 17660231 DOI: 10.1530/REP-07-0119

Nixon VL, McDougall A, Jones KT. Ca2+ oscillations and the cell cycle at fertilisation of mammalian and ascidian eggs. Biol Cell. 2000;92:187-96. PMID: 11043407 DOI: $10.1016 / S 0248-4900(00) 01068-6$

Nussey S, Whitehead S. Endocrinology: An Integrated Approach. Oxford: BIOS Scientific Publishers; 2001. PMID: 20821847 DOI: $10.4324 / 9780203450437$

Pigny $\mathrm{P}$, Jonard S, Robert $\mathrm{Y}$, Dewailly $\mathrm{D}$. Serum antiMullerian hormone as a surrogate for antral follicle count for definition of the polycystic ovary syndrome. J Clin Endocrinol Metab. 2006;91:941-5. PMID: 16368745 DOI: $10.1210 /$ jc. 2005-2076

Rajpert-De Meyts E, Jørgensen N, Graem N, Müller J, Cate $\mathrm{RL}$, Skakkebaek NE. Expression of anti-Müllerian hormone during normal and pathological gonadal development: association with differentiation of Sertoli and granulosa cells. J Clin Endocrinol Metab. 1999;84:3836-44. PMID: 10523039 DOI: $10.1210 /$ jcem.84.10.6047

Riggs R, Kimble T, Oehninger S, Bocca S, Zhao Y, Leader $B$, Stadtmauer L. Anti-Müllerian hormone serum levels predict response to controlled ovarian hyperstimulation but not embryo quality or pregnancy outcome in oocyte donation. Fertil Steril. 2011;95:410-2. PMID: 20736131 DOI: $10.1016 /$ j.fertnstert.2010.07.1042

Shebl O, Ebner T, Sommergruber M, Sir A, Tews G. Anti muellerian hormone serum levels in women with endometriosis: a case-control study. Gynecol Endocrinol. 2009;25:713-6. PMID: 19903049 DOI: $10.3109 / 09513590903159615$

Silberstein T, MacLaughlin DT, Shai I, Trimarchi JR, Lambert-Messerlian G, Seifer DB, Keefe DL, Blazar AS. Mullerian inhibiting substance levels at the time of HCG administration in IVF cycles predict both ovarian reserve and embryo morphology. Hum Reprod. 2006;21:159-63. PMID: 16123085 DOI: 10.1093/humrep/dei270 
Smeenk JM, Sweep FC, Zielhuis GA, Kremer JA, Thomas $\mathrm{CM}$, Braat DD. Antimüllerian hormone predicts ovarian responsiveness, but not embryo quality or pregnancy, after in vitro fertilization or intracyoplasmic sperm injection. Fertil Steril. 2007;87:223-6. PMID: 17081531 DOI: 10.1016/j.fertnstert.2006.06.019

Talebian S, Licciardi F, Liu M, Grifo JA, Krey LC. Assessing antimullerian hormone (AMH) as a marker of ovarian response in anonymous oocyte donors: quantity or quality? Fertil Steril. 2008;90:S267. DOI: 10.1016/j.fertnstert.2008.07.1249

Tesarík J, Kopecný V, Plachot M, Mandelbaum J. Early morphological signs of embryonic genome expression in human preimplantation development as revealed by quantitative electron microscopy. Dev Biol. 1988;128:1520. PMID: 2454852 DOI: $10.1016 / 0012-1606(88) 90261-8$

Tremellen KP, Kolo M, Gilmore A, Lekamge DN. Antimullerian hormone as a marker of ovarian reserve. Aust N Z J Obstet Gynaecol. 2005;45:20-4. PMID: 15730360 DOI: $10.1111 / \mathrm{j} .1479-828 X .2005 .00332 . x$

Van Rooij IA, Bancsi LF, Broekmans FJ, Looman CW, Habbema JD, te Velde ER. W Women older than 40 years of age and those with elevated follicle-stimulating hormone levels differ in poor response rate and embryo quality in in vitro fertilization. Fertil Steril. 2003;79:482-8. PMID: 12620425 DOI: $10.1016 /$ S0015-0282(02)04839-2 van Rooij IA, Broekmans FJ, te Velde ER, Fauser BC, Bancsi LF, de Jong FH, Themmen AP. Serum anti-Müllerian hormone levels: a novel measure of ovarian reserve. Hum Reprod. 2002;17:3065-71. PMID: 12456604 DOI: $10.1093 /$ humrep/17.12.306

Weenen C, Laven JS, Von Bergh AR, Cranfield M, Groome NP, Visser JA, Kramer P, Fauser BC, Themmen AP. AntiMüllerian hormone expression pattern in the human ovary: potential implications for initial and cyclic follicle recruitment. Mol Hum Reprod. 2004;10:77-83. PMID: 14742691 DOI: $10.1093 / \mathrm{molehr} /$ gah015

Weghofer A, Dietrich W, Barad DH, Gleicher N. Live birth chances in women with extremely low-serum antiMullerian hormone levels. Hum Reprod. 2011;26:1905-9. PMID: 21531994 DOI: 10.1093/humrep/der134

Zeuner A, Müller K, Reguszynski K, Jewgenow $K$. Apoptosis within bovine follicular cells and its effect on oocyte development during in vitro maturation. Theriogenology. 2003;59:1421-33. PMID: 12527088 DOI: $10.1016 /$ S0093-691X(02)01190-1 